\title{
Women's Health During the COVID-19 Surge in the Bronx: Reflections from Two OBGYNs on the Flatter Side of the Curve
}

\author{
Antoinette A. Danvers ${ }^{1} \cdot$ Siobhan M. Dolan ${ }^{1,2}$ (]) \\ Published online: 7 July 2020 \\ ○) Springer Science+Business Media, LLC, part of Springer Nature 2020
}

\section{Introduction}

The scariest part was the silence. In the peak of the COVID19 surge in early April, 2020, our drive times to work were cut in half; there were fewer cars on the road and the morning hustle of children going to school was gone. The bustling, vibrant borough of the Bronx was silent. It was eerie. The silence was only broken by the sound of sirens wailing down the streets as ambulances brought the severely ill COVID-19 patients to the Emergency Room. The tents went up, the ERs got busier, the ambulances kept coming, and then the morgue trucks arrived.

In our Department of Obstetrics \& Gynecology and Women's Health at Montefiore Medical Center in the Bronx, NY, we care for women in one of the most diverse boroughs in the country. With a population of over 1.4 million people, $43.6 \%$ of Bronx residents are black or African American, $56.4 \%$ are Hispanic or Latino, $35.4 \%$ are foreign born persons, and $27.3 \%$ live in poverty ("U.S. Census Bureau QuickFacts: United States," 2019). Back in early March, pausing the Bronx seemed unimaginable to us. But given the growing number of cases in New York City and the serious impact in communities of color, we knew that every moment of delay meant additional cases and deaths due to COVID-19.

In early March of 2020, before we were celebrated as front line healthcare workers in epicenter of the COVID19 pandemic, we were preparing for our battle. We wondered "when," not "if" we would become sick. We read

Siobhan M. Dolan

sdolan@montefiore.org

1 Department of Obstetrics \& Gynecology and Women's Health at Montefiore Medical Center, Albert Einstein College of Medicine, Bronx, NY, USA

2 Department of Obstetrics \& Gynecology and Women's Health at Montefiore Medical Center, Albert Einstein College of Medicine, 1695 Eastchester Road, Suite 301, Bronx, NY 10461, USA maintenance of certification articles about the novel coronavirus and attended Zoom calls on how to run a ventilator while in the backdrop we picked up our kids from college, made plans for child care and home schooling, and found ways to protect our vulnerable family members from ourselves. We left the hospital at the end of the work week on Friday March 20, 2020, knowing that our lives were about to change. On Sunday, March 22, Governor Andrew Cuomo issued the "New York State on PAUSE" Executive Order ("Governor Cuomo Signs the 'New York State on PAUSE' Executive Order," 2020) as New York City (NYC) became the epicenter of the pandemic reporting over 10,000 cases ("NYC.gov-New York City Department of Health and Mental Hygiene," 2020) with widespread community transmission of SARS-CoV-2.

As essential healthcare workers, we returned to work on Monday March 23, but the patients didn't come back. Despite the silent streets, women's health care needs continued as they could not be put on pause. We knew the time sensitive nature of clinical care for wanted and unwanted pregnancies meant our services needed to continue. While the media attention focused on care in labor \& delivery, contraceptive counseling remained critical, women were getting pregnant and seeking prenatal care, and genetic conditions and fetal anomalies were being diagnosed and terminations needed to be provided. Doing so meant working longer shifts, while we felt fear and lived in isolation. This care was needed and we provided it.

\section{Dr. Danvers from the Front Line as an OBGYN and Family Planning Specialist}

As our hospital system braced for the surge in coronavirus patients, all healthcare providers were informed about the possibility for deployment to high need areas. It was clear, regardless of how bad the increasing number of patients became, the labor \& delivery floor would remain 
covered. And while some of our OBGYN colleagues joined the medical teams caring directly for patients hospitalized with COVID-19, the labor floor remained our front line: our Emergency Room, our inpatient unit and our operating rooms. Each place demanded something different from us.

While our experience with coronavirus was new, it was reassuring to stay on the familiar territory of labor \& delivery. The first tactical decision was to staff the labor floor in cohorts, keeping the inpatient and outpatient teams separate, both to avoid the likelihood of widespread infection throughout the entire Department and to maintain outpatient providers at lower risk of infection who could always cover the labor floor as needed. In the early days of the pandemic in New York, reports about the shortage of personal protective equipment (PPE) and the lack of widespread testing elevated the concerns around healthcare worker safety.

As one of the only units in the hospital that routinely admitted otherwise healthy women, the exposure to SARSCoV-2 was often unknown. Initially, we adhered to strict testing protocols whereby rapid testing (which came back in 1-2 $\mathrm{h}$ ) was performed on patients who were symptomatic or had travelled to high risk areas. When symptoms evolved for some women during labor \& delivery, many diagnoses were only made when a post-partum fever prompted testing confirming COVID-19, after the delivering providers had already been exposed. As the pandemic evolved and testing became more widely available, we transitioned to universal testing of both patients and partners upon admission to labor $\&$ delivery, which confirmed the high rate of asymptomatic SARS-CoV-2 carriers.

The landscape of testing and PPE was changing rapidly but demand was still behind supply. Unsure of availability, we continued to conserve the PPE we had even as more became available. To minimize contact, visitors were not allowed in the hospital because each additional contact increased risk of transmission. Hence, for both their own protection as well as the protection of the staff and patients, even visitors who had lost family members to coronavirus were not allowed in the hospital. Yet, the labor \& delivery unit was unique. Previous attempts to ban visitors in other major institutions (Breslin et al. 2020) ignited strong objection among women receiving care in those institutions who expressed their fear about laboring alone. Policies were reversed by an Executive Order from New York Governor, Andrew Cuomo, requiring a partner be allowed with each laboring woman (Van Syckle and Caron 2020). In the days and weeks to follow, testing protocols on labor \& delivery expanded and we performed universal testing for all admitted patients, then later testing of both patients and their partners.

In Family Planning, we continued to provide abortion care, but the diminished access to operating rooms forced us to consider how best to care for patients in the outpatient setting. We continued to offer medication abortion and miscarriage management as well as office-based procedures whenever feasible and acceptable to the patients. We immediately transitioned to telehealth follow-up for medical management of abortion and miscarriage and for contraception counseling and management in order to limit patient interaction with the healthcare system. In many ways, keeping our doors open kept women out of the hospital at a time that it was risky for them to be there. The combination of telehealth and in-person visits kept our office-based family planning practice at almost full capacity.

One silver lining that came from the experience of working on the front line is the bond it created amongst the providers who worked together. Because the labor \& delivery coverage teams were cohorts, we worked with the same individuals for 2 weeks providing full time coverage. We built trust, provided support to each other and strategically balanced who handled coronavirus testing. In Family Planning, we learned to work with a smaller team and eventually, in combination with telehealth, saw close to as many patients as we did before the start of the pandemic. Finally, because of the history of burnout in our specialty, we benefited from having established mental health support within our Department and they actively offered counselling to those who needed it.

\section{Dr. Dolan from the Midfield as an OBGYN Geneticist and Vice Chair for Research}

Since coronavirus is a novel infection, we had no data regarding its impact on pregnancy. My early efforts in March and April focused on studying a large series of pregnancy outcomes for women with SARS-CoV-2 to examine the obstetrical experience and perinatal outcomes. Since NY was experiencing the greatest number of cases, it felt critical to collect data to inform the rest of the country. Even basic simple questions about COIVD19 in pregnancy had no answers. Were pregnant women at greater risk of becoming infected with SARS-CoV-2 and did they have a more serious clinical picture than nonpregnant women? Data out of China were limited. Working with three other major medical centers in NYC, we amassed a case series of 241 pregnancies and reported that ten required delivery due to worsening maternal respiratory status (Khoury et al. 2020) thus increasing both the preterm delivery rate and the cesarean section rate. We learned that many women were asymptomatic at presentation and developed symptoms while on labor \& delivery. And we learned that vertical transmission was unlikely with $97.5 \%$ of newborns testing negative after birth. Performing this research has helped us prepare and inform other hospitals throughout the country that: (1) universal 
testing on labor \& delivery is warranted, (2) additional ICU beds and ventilatory support will be needed for $<10 \%$ of peripartum patients, and (3) vertical transmission to the fetus is unlikely. While this data is largely reassuring, the all-important question remains: what predicts disease severity of COVID-19 and is it the same or different for pregnant women?

In the outpatient setting, patients were scared. Many questioned how could they get registered for prenatal care? And, who would see them? We turned quickly to telehealth and began doing new OB and prenatal care visits via telephone. Women came to the hospital twice in the first half of pregnancy: once at 11-13 weeks for a dating ultrasound (with nuchal translucency), vital signs, prenatal lab work and aneuploidy screening, then again at approximately 20 weeks for an anatomy ultrasound and repeat vital signs. All other visits were by telehealth-by phone in the beginning but more recently using a video application. This transition went at lightning speed but it has gone incredibly well. Early on, the patients were just so happy to hear from us because it allayed their fear and isolation. They expressed enormous appreciation. As we have settled into the new routine, video visits provide an intimacy that is interesting. As a provider, you are right there in the patient's home. It is sometimes heartbreaking, such as talking to a woman about her desire to terminate a 10 week pregnancy due to a breast cancer recurrence while her toddlers are climbing on and off of her lap. And at other times it is funny, such as asking a patient if her partner would be willing to do carrier screening and watching her yell into the next room, "Honey, the doctor wants to know if you will do generic [sic] testing." We get to see women in their bedrooms and their living rooms, with their cats and their dogs, we meet their children and their partners, and we get to see their art work and their bookshelves. It's amazing and reassuring how connected you can feel to another person when you are looking at them via a computer screen and it has allowed us to provide outstanding care.

Throughout the pause, I have been in the hospital every day. I spend most of my time on phone and video but I see the patients who need in-person care and that amounts to several patients a day. I have access to N95 masks and we have set up our offices to enforce social distancing. I show up for the patients who come in to see us because they have had a fetal demise or an anomaly diagnosed and it's particularly hard for them because they must enter the building alone. We dial in their partner or mother or sister on FaceTime and I try to offer them comfort, genetic testing options and a plan. I don't consider myself on the front line but more in the midfield. Showing up for women during this pandemic and breaking through the fear and isolation by assuring them that we will care for them has been incredibly rewarding.

\section{Conclusion}

As New York City moves out into the flatter part of the curve, we have so much to be thankful for. Most of us managed to stay healthy. Our children finished school and are getting ready for summer. Our patients continue to get the care they need and new pregnancies promise hope for the future. The weather is warming and the fear is passing. But we worry about another surge, especially since we are nowhere close to achieving herd immunity in NYC (Popovich and Sanger-Katz 2020) and a vaccine is still in the distance.

Even as we see the Bronx start to reopen, the tragic toll of COVID-19's impact on communities of color and those living in poverty remains vivid. At the time of this writing, we see the news reports of nationwide protests for equality in response to the death of George Floyd. As the New York Times reports 207,539 cases and 20,991 deaths due to coronavirus in NYC, we also see that the Bronx has two zip codes with the highest number of deaths ("New York City Coronavirus Map and Case Count," 2020). Racism and inequality deeply affect the health and well-being of our community and we will continue to work hard to effect change.

Our business school classmate, Dr. Lorna Breen, who ran the ER at New York-Presbyterian's Allen Hospital, died by suicide in April after contracting COVID-19 while caring for ill patients (Watkins et al. 2020). Thinking about her isolation and despair saddens us because we saw her on our Zoom classes but we didn't know of her pain. Her memory has highlighted the deep impact working on the front line has had on our daily lives. We remember the isolation and stress of the experience and make concerted efforts to stay connected to each other and to our families, colleagues, patients and classmates. We certainly expect that the virus will return, COVID-19 cases will surge, and the front line will again need attention. But we are reminded of the difference we hope we have made by helping each other and our patients feel connected and cared for throughout the surge. We plan to provide that attention and be there again, to care for all aspects of women's health care, as needed.

\section{References}

Breslin, N., Baptiste, C., Miller, R., et al. (2020). Coronavirus disease 2019 in pregnancy: Early lessons. American Journal of Obstetrics \& Gynecology MFM, 2(2), 100111. https://doi. org/10.1016/j.ajogmf.2020.100111.

Governor Cuomo Signs the "New York State on PAUSE" Executive Order. (2020, March 23). Retrieved May 31, 2020, from https ://www.governor.ny.gov/news/governor-cuomo-signs-new-yorkstate-pause-executive-order. 
Khoury, R., Bernstein, P., DeBolt, C., et al. (2020). Characteristics and outcomes of the first 241 births to women with SARSCoV-2 at five New York City medical centers. Obstetrics \& Gynecology. https://doi.org/10.1097/AOG.0000000000004025.

New York City Coronavirus Map and Case Count. (2020, June 2). Retrieved May 31, 2020, from https://www.nytimes.com/inter active/2020/nyregion/new-york-city-coronavirus-cases.html\#zipco de. ++

NYC.gov - The Official New York City Web Site - Redirected page. (2020). Retrieved May 31, 2020, from https://www.nyc.gov/site/ doh/covid/covid-19-main.page

Popovich, N., \& Sanger-Katz, M. (2020, May 29). The world is still far from herd immunity for coronavirus. Retrieved June 1, 2020, from https://www.nytimes.com/interactive/2020/05/28/upshot/ coronavirus-herd-immunity.html?searchResultPosition=2.++

U.S. Census Bureau QuickFacts: United States. (2019). Retrieved May 31, 2020, from https://www.census.gov/quickfacts/bronxcount ybronxboroughnewyork.++
Van Syckle, K, \& Caron, C. (2020, March 29). 'Women will not be forced to be alone when they are giving birth.' Retrieved May 31, 2020, from https://www.nytimes.com/2020/03/28/parenting/ nyc-coronavirus-hospitals-visitors-labor.html?searchResultPos ition $=1$.

Watkins, A., Rothfeld, M., Rashbaum, W., \& Rosenthal, B. (2020, April 30). Top E.R. doctor who treated virus patients dies by suicide. Retrieved May 31, 2020, from https://www.nytim es.com/2020/04/27/nyregion/new-york-city-doctor-suicide-coron avirus.html.

Publisher's Note Springer Nature remains neutral with regard to jurisdictional claims in published maps and institutional affiliations. 